\title{
Original
}

\section{Measurement of Post-Treatment Changes in Brain Metabolites in Patients with Generalized Anxiety Disorder using Magnetic Resonance Spectroscopy}

\author{
Hossein Mohammadi ${ }^{\oplus}$, Vahid Changizi*๑ ${ }^{2 *}$, Nader Riyahi Alam³, \\ Fatemeh Rahiminejad ${ }^{4}$, Mehdi Soleimani5, Afsaneh Qardashi ${ }^{6}$
}

\begin{abstract}
Background: From previous studies, we know the correlations of some brain metabolites with a generalized anxiety disorder (GAD) and its symptoms. The response of GAD patients to various treatments is not the same and finding the best treatment option for each patient takes a long period of time.

Objective: In this study, we try to examine if there is any relationship between a special treatment option and GAD patients' response and brain metabolite correlation with anxiety level change.

Material and Methods: This study is a clinical trial type of studies. We have used proton MRS (1H-MRS) with field strength of 3 Tesla to assess whether a different treatment option makes different responses based on metabolite changes. We chose 16 patients based on Hamilton's anxiety rate and a psychiatrist diagnosis. Patients were divided into two groups randomly. Each group took different treatments. Before treatment started, patients underwent MRS imaging and 8 weeks after treatment as well. Our study lacked a control group, and the results were analyzed by comparing the measured values of metabolites and clinical scores before and after treatment.
\end{abstract}

Results: The NAA and Cho concentration increased after treatments and $\mathrm{Cr}$ concentration remained constant in both groups. Both groups showed improvements in their symptoms of anxiety and also in their clinical score rates. Sertraline group showed a more increase in NAA concentration than $\mathrm{CBT}$ and also a more decrease in HAMA and HAMD-17 scores.

Conclusion: A simultaneously increase in NAA and Cho in both groups and a decrease in clinical anxiety levels demonstrate that NAA and Cho concentration are associated negatively with anxiety levels. In addition, both CBT and sertraline are effective in the improvement of anxiety symptoms.

Citation: Mohammadi H, Changizi V, Riyahi Alam N, Rahiminejad F, Soleimani M, Qardashi A. Measurement of Post-Treatment Changes in Brain Metabolites in Patients with Generalized Anxiety Disorder using Magnetic Resonance Spectroscopy. J Biomed Phys Eng. 2022;12(1):51-60.

\section{Keywords}

Magnetic Resonance Spectroscopy; Generalized Anxiety Disorder; Sertraline; Cognitive Behavior Therapy; Dorsolateral Prefrontal Cortex; Brain Metabolites

\section{Introduction}

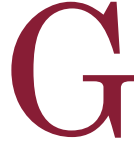
eneralized anxiety disorder prevalence is about 6 percent in the population [1]. It affects the quality of daily life of involved person via mood disorders like uncontrollable anxiety and psychiatric distress [2]. The rate of GAD comorbidity with other psychological disorders is high, and this causes different symptoms in patients, which
${ }^{1} \mathrm{MSc}$, Department of Medical Physics and Biomedical Engineering, School of Medicine, Tehran University of Medical Sciences, Tehran, Iran 2PhD, Department of Technology of Radiol ogy and Radiotherapy, Allied Medical Sciences School, Tehran University of Medical Sciences, Tehran, Iran

${ }^{3} \mathrm{PhD}$, Department of Medical Physics and Biomedical Engineering, School of Medicine, Tehran University of Medical Sciences, Tehran, Iran

${ }^{4} \mathrm{MD}$, PhD, Department of Psychiatry, Tehran University of Medical Sciences, Tehran, Iran

${ }^{5} \mathrm{PhD}$, Department of Psychiatry, Tehran University of Medical Sciences, Tehran, Iran

${ }^{6} \mathrm{BSc}$, Department of Radiology, Valiasr Hospita of Meshgin Shahr, Ardabil, Iran

*Corresponding author: Vahid Changizi

Department of Technology of Radiology and Radiotherapy, Allied Medical Sciences School, Tehran University of Medical Sciences, Tehran, Iran E-mail: changizi@tums. ac.ir

Received: 23 July 2019 Accepted: 19 August 2019 
these existing different symptoms in patients lead into difficult diagnosis [3]. GAD shows comorbidity with panic disorder, depression [3]. Anxiety disorder causes emotional problems such as low tolerance level and unsuppressed anger and also memory impairment $[4,5]$. It has a chronic period if not well treated [6]. For years or decades, GAD patients may suffer from their disorders [7]. The suicide rate in GAD patients is higher than other anxiety disorders $(2.3 \%)$ [3]. The neurology is unreliable for the theoretical survey [8]. GAD causes increased family burden and job disability $[9,10]$. The basic GAD processes may also be the cause of other anxiety disorders [11]. GAD patient's response is not good for each therapeutic approach and finding the best treatment option for each patient takes a long time [12]. Selective serotonin reuptake inhibitor (SSRIs) and serotonin and noradrenaline reuptake (SNRIs) medications which are used to treat GAD [13] cause some problems such as nausea and sexual dysfunction and also at the beginning of the treatment make anxiety symptoms worse [14].

Glutamate modulating materials because of reducing the toxicity of glutamate and improving the hippocampal nerves disorder may be effective for GAD patients. Recognizing the biological factors of the GAD could be helpful to understand the neurological mechanism and symptomatic change in patients. There are studies that have been identified the correlated metabolites with GAD. One study on dorsolateral prefrontal cortical pathology has specified that GAD with asymmetrical increases in the ratio of N-Acetylaspartate/creatine correlated [15]. Increasing hippocampal N-Acetylaspartate (NAA) concentration positively is associated with improvement of anxiety symptoms [16]. Symptom severity in GAD patients correlated with low choline/NAA ratio in the dorsolateral prefrontal cortex (DLPFC) of brain [17]. Comparison between treatment options and brain metabolites changes due to the treatment used may be helpful to know which treatment works better or effect faster. This comparison could even demonstrate if given treatment correlated with different metabolites or all treatments correlated with the same metabolites. Magnetic resonance spectroscopy is a medical imaging modality that is widely used for neuroimaging to assess metabolites concentration in the different regions of the brain [18]. We used H-MRS to assess the metabolite concentrations in DLPFC before and after treatment in GAD patients to see differences between cognitive behavior therapy (CBT) and treatment with sertraline.

\section{Material and Methods}

\section{Subjects}

This study is a clinical trial type of studies. Sixteen GAD patients (mean age 26.15 years) which none of them had pure GAD but all of them had comorbidity with other psychiatric disorders, mostly depression were entered in this study. Patients were diagnosed using DSM-IV-TR and psychiatrist examination. To determine the patient anxiety level, patients answered three sets of questions, including generalized anxiety disorder scale 7 (GAD7) with cut off score $>4$, Hamilton anxiety rating scale (HAMA) with cut off score $>14$ and Hamilton rating scale for depression 17 (HAMD-17) with cut off score $>7$. All patients were diagnosed among students of Tehran University of Medical Sciences and had no history of psychological treatments. Patients were randomly divided into two groups with different treatments. One group received sertraline and another one was treated with CBT. Before treatment started, patients underwent MRS imaging. Then each group was treated for 8 weeks with only one of the therapeutic approaches as stated above. One group was treated with sertraline so that each patient received one dose of $100 \mathrm{mg}$ per day. Each member of the group, treated with CBT, had treatment sessions twice a week, and each session was $45 \mathrm{~min}$. After the termination of the 
Measurement of Brain Metabolites by MRS

treatment period, each patient was reimaged with MRS. In this way, we could compare the results before and after treatment. Our study lacked a control group, and the results were analyzed by comparing the measured values of metabolites and clinical scores before and after treatment. Imaging protocols were performed at the national brain-mapping laboratory. All patients signed the consent form before entering the study. Table 1 summarized the demographic characteristics of patients participated in this study.

\section{Neuroimaging procedures}

Imaging examinations from subjects were performed on a 3.0 Tesla Siemens MR scanner (SIEMENS MAGNETOM Prisma). The high resolution structural $\mathrm{T} 1$ weighted fast spin echo sequences (TR/TE 2000/3.5 ms, slice thickness 1 millimeter, matrix size $=256 \times 256$ and field of view $=22 \times 22 \mathrm{~cm}$ ) was imple- mented on axial plane, then reconstructions in coronal and sagittal planes were carried out. Using structural images we localized single voxel MR spectroscopy in the right and left DLPFC. (Figure 1) DLPFC is one of the main associated regions with GAD patient's cognitive process and emotional regulations $[3,19]$. In addition, a single voxel MRS imaging was proceeded using PRESS sequence with $8 \mathrm{~cm}^{3}$ voxel size $\left(20 \times 20 \times 20 \mathrm{~mm}^{3}\right)$, a field of view= $22 \times 22 \mathrm{~cm}^{2}$, data points (vector size $=1024$, 96 acquisitions and TR/TE 2000/30). Before the spectral measurements starts, $\mathrm{B}_{0}$ magnetic field shimming was carried out and water suppression was performed using chemical shift selective saturation pulses with $50 \mathrm{HZ}$ bandwidth.

\section{Postprocessing of MRS spectrum}

Data processing started after completing the data acquisition, row data with rda for-

Table 1: Demographic characteristics and mean clinical questionnaire scores of patients before treatment

\begin{tabular}{ccccccc} 
& Sample size & Age(years) & Sex(male/female) & $\begin{array}{c}\text { GAD-7 } \\
\text { Score }\end{array}$ & $\begin{array}{c}\text { HAMA } \\
\text { score }\end{array}$ & $\begin{array}{c}\text { HAMD-17 } \\
\text { score }\end{array}$ \\
\hline Sertraline & 8 & 26.5 & $8 / 0$ & 8.62 & 22.87 & 13.5 \\
\hline CBT & 8 & 25.8 & $7 / 1$ & 9.37 & 22 & 9.4
\end{tabular}

HAMA: Hamilton Anxiety Rating Scale, cut-off score >14; GAD-7: Generalized Anxiety Disorder Scale-7, cut-off score >4; HAMD-17: Hamilton Rating Scale for Depression-17, cut-off score >7, CBT: Cognitive Behavior Therapy

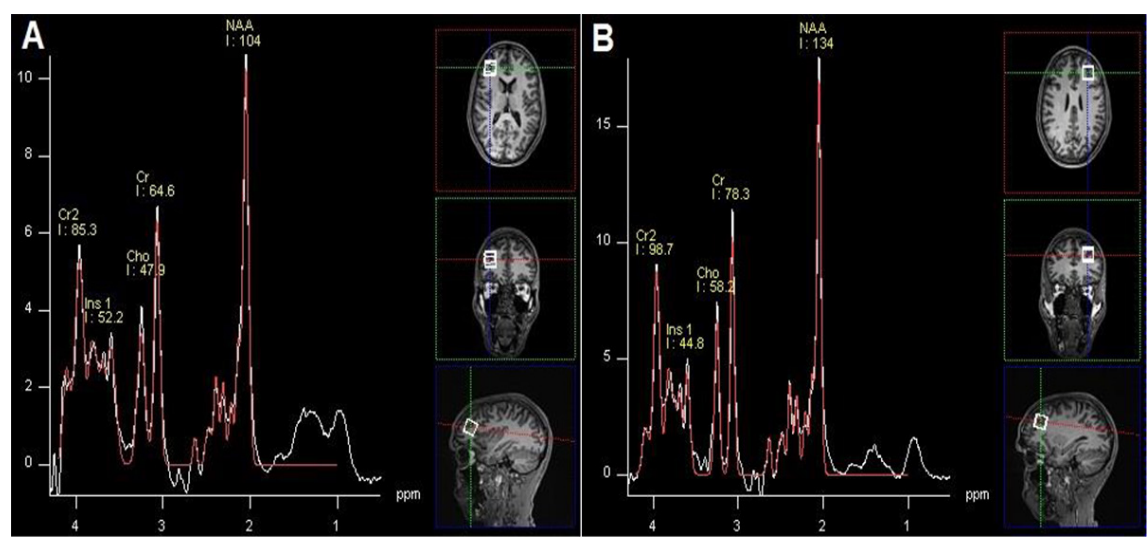

Figure 1: A) localized voxel and it's a spectrum in the right dorsolateral prefrontal cortex (DLPFC) of the patient's brain, B) localized voxel and it's a spectrum in the left DLPFC of the same patient's brain. 
mat were processed using JMRU software. In the first step phase correction accomplished by specifying set reference for water peak at $4.7 \mathrm{ppm}$. Then water peak removed using the SVD filter (Figure 2A). After that apodization was performed with $2 \mathrm{HZ}$ Lorentzian filter to reduce the noise effect on the spectrum and made it smoother. We used AMRES (advanced method for accurate, robust and efficient spectral fitting) package to obtain metabolite peaks (Figure 2B). Metabolite concentrations were calculated [20, 21, 22] following and we enter the chemical shifts of NAA, Cr and Cho (2.02 ppm, $3.03 \mathrm{ppm}$, and $3.22 \mathrm{ppm}$ respectively) at prior knowledge for spectrums.

\section{Statistical analysis}

Statistical analysis of data was performed using SPSS software (IBM SPSS statistics 19). First, we used the Kolmogorov-Smirnova test to verify the normal distribution of data and homogeneity of their variance. Then independent t-test with a $95 \%$ confidence interval and significance at $\mathrm{P}<0.05$ was carried out to compare the metabolite concentrations and their ratio. We compared the concentrations of metabolites between the two groups of patients.
This comparison was made both by comparing the absolute concentration of metabolites and the ratio of the concentration of metabolites (Cho/NAA, Cr/NAA and Cho/Cr) before and after treatment. We assumed that right and left DLPFC and their metabolites have no interaction. We also used a paired sample test to compare metabolites change in left and right DLPFC in both CBT and sertraline group and also their clinical scores changes after treatment (Table 2). All results in Table 2 demonstrate paired variables because their value indicates the difference in post-treatment values from pre-treatment values.

\section{Results}

Treatment cause metabolite alteration in both groups. NAA and Cho changes were more than $\mathrm{Cr}$ and all of them have shown an increase in their concentrations after treatment. Although $\mathrm{Cr}$ concentration increased after treatment, its increase is not significant and in comparison to NAA and Cho it was almost constant. Table 2 shows the result of the paired sample t-test between right and left DLPFC in our groups. Two of the patients in the sertraline group experienced worse symp-

residue
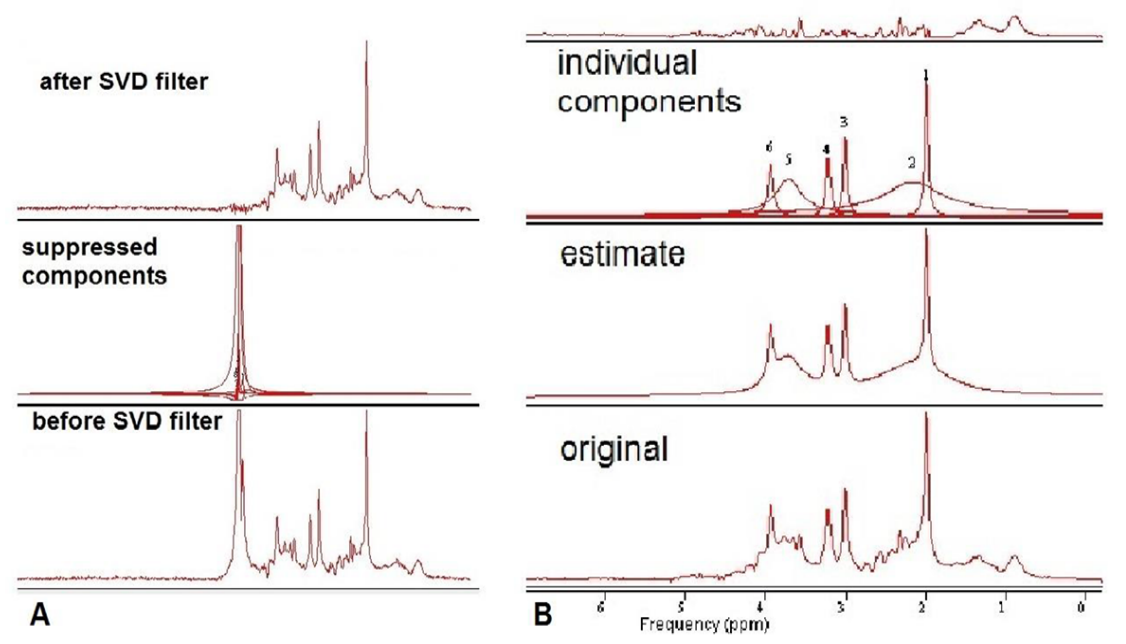

Figure 2: A) The result of using the SVD filter to remove the water peak, B) The result of using AMARIS filter to calculate the concentrations of the metabolites. The concentration of each metabolite is calculated as the integral of the sub-level of that metabolite peak. 
Table 2: Paired sample t-test

\begin{tabular}{ccccccccc}
\multicolumn{9}{c}{${ }^{1}$ Sertraline } \\
\hline Variable & ${ }^{3}$ Mean \pm SD & d.f & t-value & p-value & ${ }^{3}$ Mean \pm SD & d.f & t-value & p-value \\
\hline Left NAA & $48.723 \pm 31.020$ & 7 & 4.443 & 0.003 & $25.902 \pm 32.070$ & 7 & 2.284 & 0.056 \\
\hline Right NAA & $44.573 \pm 30.320$ & 7 & 4.158 & 0.004 & $14.583 \pm 33.250$ & 7 & 1.241 & 0.255 \\
\hline Left Cr & $1.368 \pm 7.050$ & 7 & 0.549 & 0.600 & $0.863 \pm 10.759$ & 7 & 0.227 & 0.827 \\
\hline Right Cr & $0.417 \pm 7.029$ & 7 & 0.168 & 0.871 & $2.643 \pm 10.277$ & 7 & 0.728 & 0.490 \\
\hline Left Cho & $9.643 \pm 9.853$ & 7 & 2.768 & 0.028 & $9.951 \pm 4.701$ & 7 & 5.987 & 0.001 \\
\hline Right Cho & $9.485 \pm 10.896$ & 7 & 2.462 & 0.043 & $14.633 \pm 8.274$ & 7 & 5.002 & 0.002 \\
\hline HAMA & $-4.125 \pm 3.044$ & 7 & -3.832 & 0.006 & $-2.875 \pm 2.850$ & 7 & -2.853 & 0.025 \\
\hline GAD-7 & $-1.250 \pm 2.375$ & 7 & -1.488 & 0.180 & $-1.875 \pm 1.552$ & 7 & -3.416 & 0.011 \\
\hline HAMD-17 & $-2.50 \pm 2.070$ & 7 & -3.416 & 0.011 & $-1.000 \pm 3.295$ & 7 & -0.858 & 0.419
\end{tabular}

${ }^{1}$ The group treated with sertraline, ${ }^{2}$ The group treated with cognitive behavior therapy, ${ }^{3}$ The mean and SD calculated by mean concentration of each metabolite after treatment minus from the concentration of same metabolite before treatment. For example: $\mathrm{Cr}$ mean and $\mathrm{SD}=\mathrm{Cr}$ after treatment $-\mathrm{Cr}$ before treatment. NAA: N-Acetylaspartate, HAMA: Hamilton Anxiety Rating Scale, GAD-7: Generalized Anxiety Disorder Scale-7, HAMD-17: Hamilton Rating Scale for Depression-17

toms after treatment. The rest of the patients showed improvement in their symptoms severity. After treatment, the mean metabolite concentrations change in sertraline group increased as follows: NAA, $\mathrm{Cr}$ and Cho increased in the left DLPFC $12.55 \%, 0.6 \%$, and $5.44 \%$, respectively and in the right DLPFC, $9.96 \%, 0.21 \%$ and $6.5 \%$, respectively. The clinical questionnaire scores decreased as follows: $\mathrm{HAMA}=18.03 \%$, GAD- $7=14.5 \%$ and HAMD- $17=18.5 \%$. In CBT group, in the left DLPFC NAA, Cr and Cho increased, respectively $6.16 \%, 0.35 \%$ and $5.55 \%$. In the right DLPFC, the increase in metabolite concentration was as follows: $\mathrm{NAA}=2.89 \%, \mathrm{Cr}=0.98 \%$ and $\mathrm{Cho}=8.72 \%$ and the decrease in clinical scores of CBT group is as follows: HAMA= $13.06 \%$, GAD-7 $=20.01 \%$ and HAMD-17= $8.24 \%$.

Figure 3, 4 and 5 shows a graphical representation of results in both groups.

\section{Discussion}

${ }^{1} \mathrm{H}-\mathrm{MRS}$ imaging privides the ability to measure the changes of brain metabolites concentrations related to axonal damage status and axonal pathogenesis and also neuronal loss
[23]. The NAA concentration in both left and right DLPFC and in both groups increased after treatment. Although the increase in NAA in the sertraline group is more than the CBT group, their increase pattern is similar, (Figure 3I). Following an increase in NAA, patients symptoms and clinical scores decreased (Figure 5). Therefore, there is an inverse relationship between NAA concentration in the left and right DLPFC of brain and patient's symptoms severity. The same result has been observed due to an increase in hippocampal NAA concentration [16]. Sanjay J. Mathew et al. [15] demonstrated previously in their study on DLPFC cortical pathology that the NAA/ $\mathrm{Cr}$ ratio, which is a measure of neuronal viability, increased in the DLPFC in GAD patients. This finding is also consistent with our study. Consistent with the present study NAA/ $\mathrm{Cr}$ concentration increased in the right DLPFC [16]. In another study carried out by ChungMan Moon et al. [17], the concentration of NAA in patients with GAD remains constant, contrary to our study. Based on study carried out by Chadi G. Abdallah et al. [24], the hippocampus volume reduced in GAD patients but patients, after treated with riluzole, have 

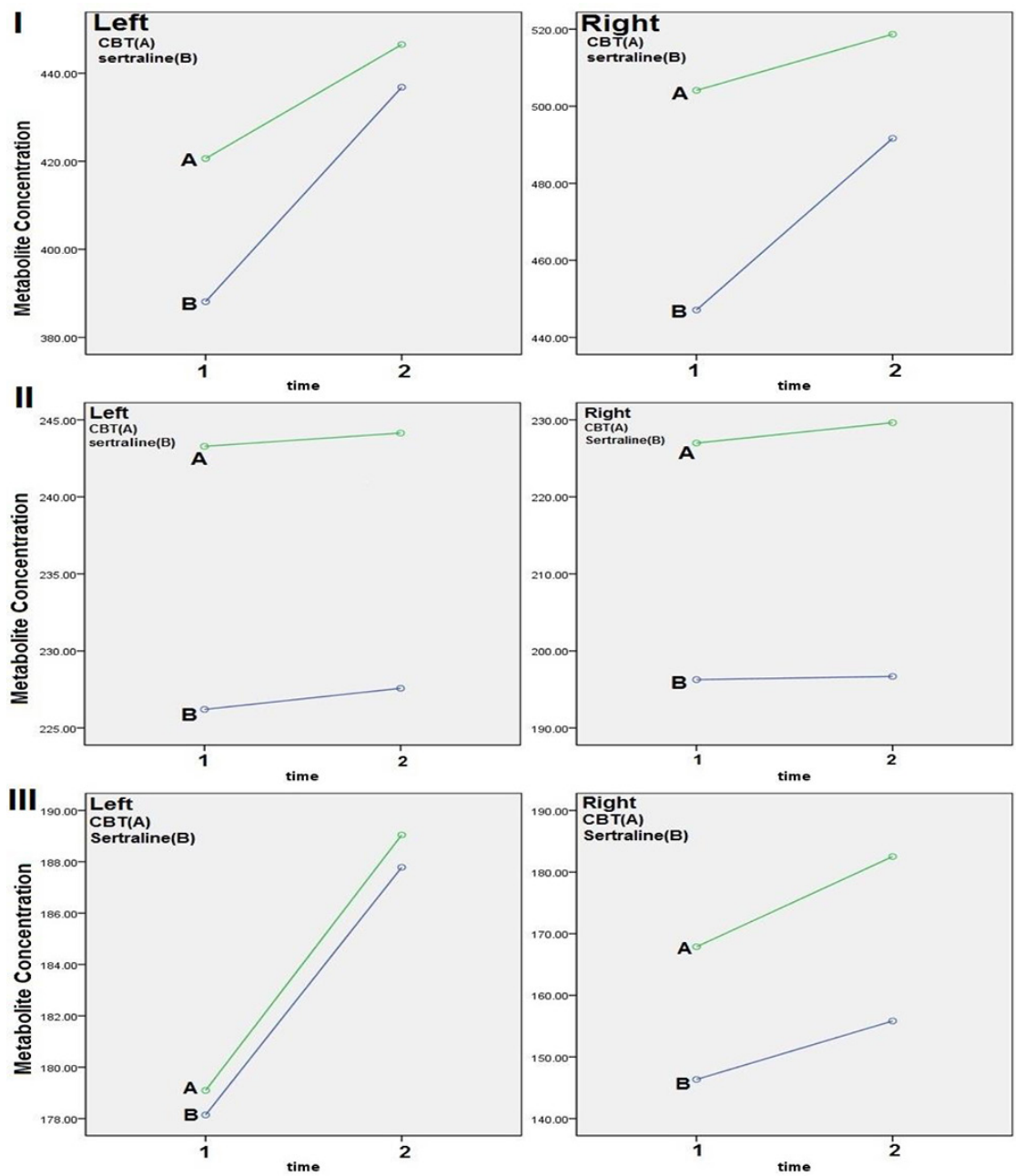

Figure 3: I) N-Acetylaspartate (NAA) concentration, II) $\mathrm{Cr}$ concentration and III) Cho concentration in left and right dorsolateral prefrontal cortex (DLPFC) before treatment 1 and after treatment 2 . The vertical axis indicates the concentration of the metabolite and the numbers 1 and 2 on the horizontal axis indicate MRS imaging from patients before and after treatment, respectively. The letters $A$ and $B$ specify cognitive behavior therapy (CBT) and sertraline groups, respectively.

shown an increase in hippocampus volume and simultaneously the increase in NAA concentration was observed.

The concentration of creatine has increased slightly, which can be said remains constant so none of the treatment has an effect on its change (Figure 3II). Cr stores energy in cells as high-energy phosphate stores. $\mathrm{Cr}$ through its buffering role for adenosine triphosphate and providing energy from it, protects tissue against hypoxia damage. $\mathrm{Cr}$ also can simultaneously cause neuronal protection against glutamate toxicity and $\beta$-amyloid [25]. Under normal and stress condition $\mathrm{Cr}$ and its supplements increase brain function [26]. Most previous studies have stated that $\mathrm{Cr}$ altered in mental disorders for example in bipolar disorder [27], depressive disorder [28] and panic disorder [29]: thus, metabolite/Cr concentration ratio may cause misinterpretation [30]. However, these studies usually examined the differences between patients with these disorders and healthy subjects, we examined the effect of treatment on metabolites concentration 

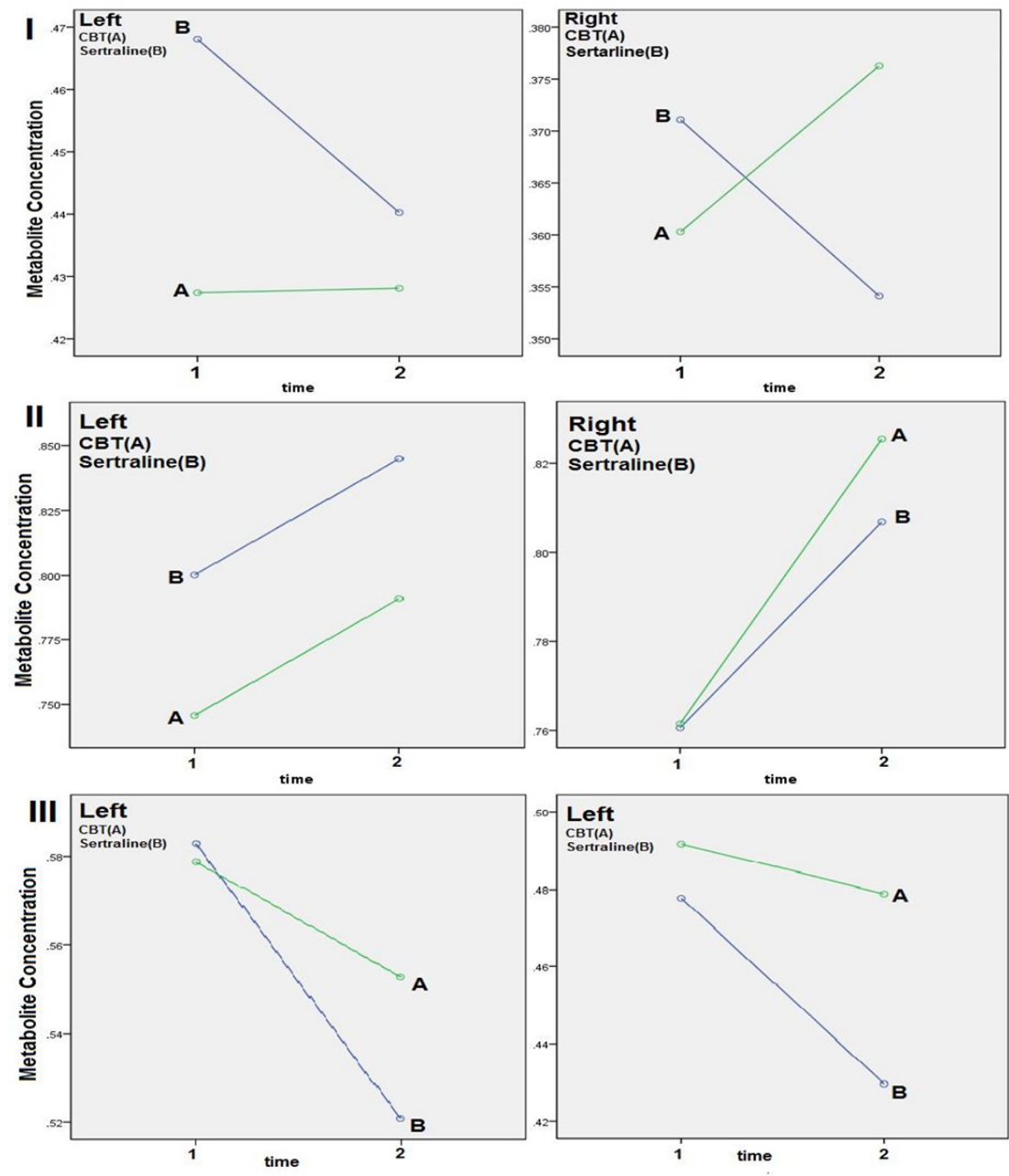

Figure 4: I) Cho/NAA, II: Cho/Cr and III) Cr/NAA concentration ratio before treatment (1) and after treatment (2). The vertical axis indicates the metabolite concentration ratio. The letters $A$ and B specify cognitive behavior therapy (CBT) and sertraline groups respectively.
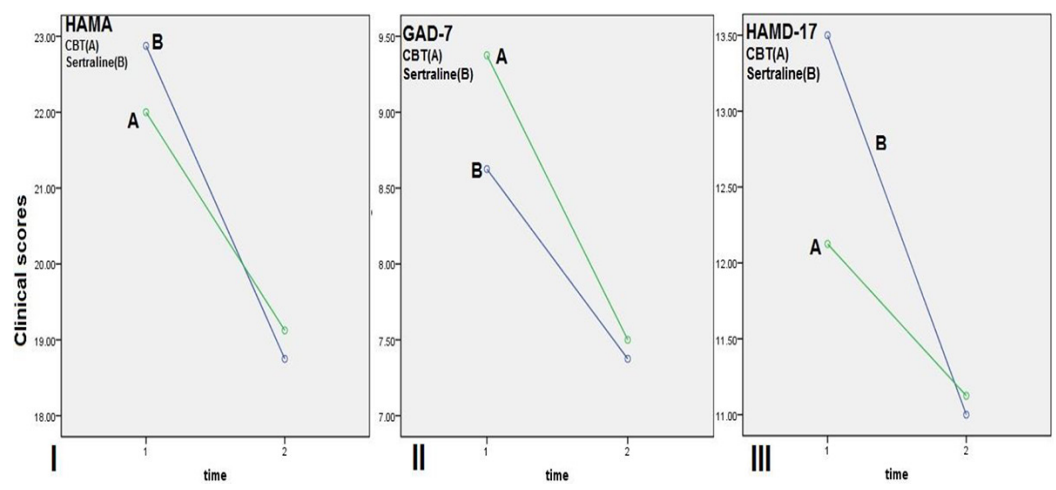

Figure 5: I) Hamilton anxiety rating scale scores, II) generalized anxiety disorder scale 7 scores and III) Hamilton rating scale for depression 17 scores in cognitive behavior therapy (CBT) group (A) and sertraline group (B) before treatment (1) and after treatment (2). 
alteration only in GAD patients and healthy subjects participated in our study. Thus, the present study states that in GAD patients treated with sertraline and CBT, metabolite/ $\mathrm{Cr}$ concentration ratio may be reliable due to the constant $\mathrm{Cr}$ concentration. In contrary to our finding, in the patient with border line personality disorder (BPD), the Cr concentration decreased in the left amygdala [28].

As noted in our study, the Cho concentration in both left and right DLPFC increased after treatments (Figure 3). Therefore, the $\mathrm{Cho} / \mathrm{Cr}$ ratio has also increased due to the constant concentration of $\mathrm{Cr}$ (Figure 4). Simultaneously, with increase in Cho concentration, the anxiety levels decreased. In the synthesis of acetylcholine used in cholinergic neurotransmission, Cho is an essential element [17]. Brain development, human cognitive processes, memory, and learning are correlated with Cho [31, 32]. Finding of a previous study [17] indicated that an increase in the Cho concentration level correlated with a decrease of anxiety levels which is in consistent with our study. Moreover, in Ferguson et al. study, a positive correlation demonstrated between the level of Cho concentration and cognitive functions [33]. The present study shows both treatments cause an increase in Cho concentration so that both are effective in cognitive functions improvement and decrease of anxiety level.

As is clear from the above statements, our study results are consistent with some studies and contradict with others. Therefore, there are many contradictions between the findings of previous studies with each other as well. To overcome these ambiguities, more studies are needed for anxiety disorders. The present study compared the efficacy of two different treatments in GAD patients simultaneously in one particular region of brain. Sertraline is more effective in increasing NAA than CBT (Figure 3). However, in other metabolites, there are no significant differences between these groups. NAA and Cho both increased in groups but their ratio has changed in the op- posite direction, especially in the right DLPFC (Figure 4). Therefore, their ratio is unreliable for interpretation. Another interesting finding is that both treatments caused improvement in anxiety symptoms and decrease in anxiety levels although sertraline caused more decrease in HAMA and HAMD-17 than CBT (Figure 5), and sertraline impact is greater. It is important to note that CBT has no pharmaceutical side effect and also unlike sertraline dose not worsen the anxiety symptoms in some patients. Therefore, researches on other treatments and drug treatments may be helpful for GAD patients. Some of the limitations in this study are the small sample size ( 8 participate in each group) and lack of control group.

\section{Conclusion}

This study suggests that NAA and Cho concentration associated negatively with anxiety levels in GAD patients and treatment with sertraline and cognitive behavior therapy both are effective in improving the symptoms of the anxiety. Future studies can measure the effectiveness of both treatments simultaneously and compare them with when we use only one treatment.

\section{Acknowledgment}

This study has been supported by Tehran University of medical sciences Grant number: 36935, Roozbeh Hospital of Tehran University of medical sciences, Tehran University counseling center, and national brain mapping laboratory.

\section{Authors' Contribution}

Vahid Changizi and Nader Riyahi Alam conceived the idea. The introduction of the paper was written by Hossein Mohammadi and Nader Riyahi Alam. Hossein Mohammadi and Afsaneh Qardashi gather the images and the related literature and also help with the writing of the related works. The method implementation was carried out by Hossein Mohammadi and Afsaneh Qardashi. Diagnosis and treatment of GAD patients carried out by Fatemeh Rahiminejad and Mehdi Soleymani. Results and Analysis were carried out by Mehdi Soleymani, Hossein Mohammadi, and 
Vahid Changizi. The research work was proofread and supervised by Vahid Changizi and Nader Riyahi Alam. All the authors read, modified, and approved the final version of the manuscript.

\section{Ethical Approval}

The Ethics Committee of Tehran University of Medical Sciences approved the protocol of the study (Ethic cod: IR.TUMS.MEDICINE. REC.1397.436).

\section{Informed consent}

All patients signed the consent form before entering the study.

\section{Funding}

This study has been supported by Tehran University of medical sciences Grant number: 36935.

\section{Conflict of Interest}

None

\section{References}

1. Wittchen HU, Jacobi F. Size and burden of mental disorders in Europe-a critical review and appraisal of 27 studies. European Neuropsychopharmacology. 2005;15(4):357-76. doi: 10.1016/j.euroneuro.2005.04.012. PubMed PMID: 15961293.

2. American Psychiatric Association. Diagnostic and Statistical Manual of Mental Disorders, Fourth Edition, Text Revision I Behavenet. [Accessed: 03Jul-2019]. Available from: https://behavenet.com/ diagnostic-and-statistical-manual-mental-disordersfourth-edition-text-revision.

3. Bernal M, Haro JM, Bernert S, Brugha T, et al. Risk factors for suicidality in Europe: results from the ESEMED study. Journal of Affective Disorders. 2007;101(1-3):27-34. doi: 10.1016/j. jad.2006.09.018. PubMed PMID: 17074395.

4. Roemer L, Lee JK, Salters-Pedneault K, et al. Mindfulness and emotion regulation difficulties in generalized anxiety disorder: Preliminary evidence for independent and overlapping contributions. Behavior Therapy. 2009;40(2):142-54. doi: 10.1016/j. beth.2008.04.001. PubMed PMID: 19433145. PubMed PMCID: PMC3719394.

5. Mantella RC, Butters MA, Dew MA, Mulsant BH, et al. Cognitive impairment in late-life generalized anxiety disorder. The American Journal of Geriatric Psychiatry. 2007;15(8):673-9. doi: 10.1097/ JGP.0b013e31803111f2. PubMed PMID: 17426260.
6. Maron E, Nutt D. Biological Markers of Generalized Anxiety Disorder. Focus. 2018;16(2):210-8. doi: 10.1176/appi.focus.16205. PubMed PMID: 32015708. PubMed PMCID: PMC6526851.

7. Bandelow B, Michaelis S. Epidemiology of anxiety disorders in the 21st century. Dialogues in Clinical Neuroscience. 2015;17(3):327. PubMed PMID: 26487813. PubMed PMCID: PMC4610617.

8. Drevets WC, Price JL, Furey ML. Brain structural and functional abnormalities in mood disorders: implications for neurocircuitry models of depression. Brain Structure and Function. 2008;213(1-2):93118. doi: 10.1007/s00429-008-0189-x. PubMed PMID: 18704495. PubMed PMCID: PMC2522333.

9. Ahola K, Virtanen M, Honkonen T, et al. Common mental disorders and subsequent work disability: a population-based Health 2000 Study. Journal of Affective Disorders. 2011;134(1-3):365-72. doi: 10.1016/j.jad.2011.05.028. PubMed PMID: 21664696.

10. Wedegaertner F, Arnhold-Kerri S, Sittaro NA, et al. Depression-and anxiety-related sick leave and the risk of permanent disability and mortality in the working population in Germany: a cohort study. BMC Public Health. 2013;13(1):145. doi: 10.1186/14712458-13-145. PubMed PMID: 23413800. PubMed PMCID: PMC3698165.

11. Hazlett-Stevens H. Psychological approaches to generalized anxiety disorder: A clinician's guide to assessment and treatment. Springer; 2008.

12. Shin LM, Davis FC, VanElzakker MB, et al. Neuroimaging predictors of treatment response in anxiety disorders. Biology of Mood \& Anxiety Disorders. 2013;3(1):15. doi: 10.1186/2045-5380-315. PubMed PMID: 23915782. PubMed PMCID: PMC3750275.

13. Bandelow B, Zohar J, Hollander E, et al. World Federation of Societies of Biological Psychiatry (WFSBP) guidelines for the pharmacological treatment of anxiety, obsessive-compulsive and posttraumatic stress disorders-first revision. The World Journal of Biological Psychiatry. 2008;9(4):248312. doi: $10.1080 / 15622970802465807$. PubMed PMID: 18949648.

14. Buoli M, Caldiroli A, Caletti E, Paoli RA, Altamura AC. New approaches to the pharmacological management of generalized anxiety disorder. Expert Opinion on Pharmacotherapy. 2013;14(2):175-84. doi: $10.1517 / 14656566.2013 .759559$. PubMed PMID: 23282069.

15. Mathew SJ, Mao X, Coplan JD, Smith EL, et al. Dorsolateral prefrontal cortical pathology in generalized anxiety disorder: a proton magnetic resonance spectroscopic imaging study. American Journal of 
Psychiatry. 2004;161(6):1119-21. doi: 10.1176/ appi.ajp.161.6.1119. PubMed PMID: 15169704.

16. Mathew SJ, Price RB, Mao X, Smith EL, et al. Hippocampal $\mathrm{N}$-acetylaspartate concentration and response to riluzole in generalized anxiety disorder. Biological Psychiatry. 2008;63(9):891-8. doi: 10.1016/j.biopsych.2007.09.012. PubMed PMID: 18028881. PubMed PMCID: PMC2385784.

17. Moon CM, Kang HK, Jeong GW. Metabolic change in the right dorsolateral prefrontal cortex and its correlation with symptom severity in patients with generalized anxiety disorder: Proton magnetic resonance spectroscopy at $3 \mathrm{~T}$ esla. Psychiatry and Clinical Neurosciences. 2015;69(7):422-30. doi: 10.1111/pcn.12279. PubMed PMID: 25611853.

18. Lyoo IK, Renshaw PF. Magnetic resonance spectroscopy: current and future applications in psychiatric research. Biological Psychiatry. 2002;51(3):195207. doi: 10.1016/s0006-3223(01)01313-0. PubMed PMID: 11839362.

19. Price RB, Eldreth DA, Mohlman J. Deficient prefrontal attentional control in late-life generalized anxiety disorder: an fMRI investigation. Translational Psychiatry. 2011;1(10):e46. doi: 10.1038/ tp.2011.46. PubMed PMID: 22833192. PubMed PMCID: PMC3309492.

20. Barker PB, Soher BJ, Blackband SJ, et al. Quantitation of proton NMR spectra of the human brain using tissue water as an internal concentration reference. NMR in Biomedicine. 1993;6(1):89-94. doi: 10.1002/nbm.1940060114. PubMed PMID: 8384470.

21. Helms G. Analysis of 1.5 Tesla proton MR spectra of human brain using LCModel and an imported basis set. Magnetic Resonance Imaging. 1999;17(8):12118. doi: 10.1016/s0730-725x(99)00058-2. PubMed PMID: 10499683.

22. Christiansen $P$, Henriksen 0 , Stubgaard M, Gideon $P$, Larsson HB. In vivo quantification of brain metabolites by $1 \mathrm{H}-\mathrm{MRS}$ using water as an internal standard. Magnetic Resonance Imaging. 1993;11(1):10718. doi: 10.1016/0730-725x(93)90418-d. PubMed PMID: 8423713.

23. Meyrhoff DJ, Mackay S, Constans JM, Norman $D$, et al. Axonal injury and membrane alterations in Alzheimer's disease suggested by in vivo proton magnetic resonance spectroscopic imaging. Annals of Neurology. 1994;36(1):40-7. doi: 10.1002/ ana. 410360110.

24. Abdallah CG, Coplan JD, Jackowski A, et al. A pilot study of hippocampal volume and $\mathrm{N}$-acetylaspartate (NAA) as response biomarkers in riluzole-treated patients with GAD. European Neuropsychopharmacology. 2013;23(4):276-84. doi: 10.1016/j.eu- roneuro.2012.05.009. PubMed PMID: 22739126. PubMed PMCID: PMC3473175.

25. Brewer GJ, Wallimann TW. Protective effect of the energy precursor creatine against toxicity of glutamate and $\beta$-amyloid in rat hippocampal neurons. Journal of Neurochemistry. 2000;74(5):196878. doi: 10.1046/j.1471-4159.2000.0741968.x. PubMed PMID: 10800940.

26. Andres RH, Ducray AD, Schlattner U, et al. Functions and effects of creatine in the central nervous system. Brain Research Bulletin. 2008;76(4):329-43. doi: $10.1016 /$ j.brainresbull.2008.02.035. PubMed PMID: 18502307.

27. New AS, Hazlett EA, Newmark RE, et al. Laboratory induced aggression: a positron emission tomography study of aggressive individuals with borderline personality disorder. Biological Psychiatry. 2009;66(12):1107-14. doi: 10.1016/j.biopsych.2009.07.015. PubMed PMID: 19748078. PubMed PMCID: PMC2788117.

28. Hoerst M, Weber-Fahr W, Tunc-Skarka N, et al. Metabolic alterations in the amygdala in borderline personality disorder: a proton magnetic resonance spectroscopy study. Biological Psychiatry. 2010;67(5):399-405. doi: 10.1016/j.biopsych.2009.09.030. PubMed PMID: 19931853.

29. Gemar MC, Segal ZV, Mayberg HS, et al. Changes in regional cerebral blood flow following mood challenge in drug-free, remitted patients with unipolar depression. Depression and Anxiety. 2007;24(8):597-601. PubMed PMID: 17133439. doi: $10.1002 /$ da.20242.

30. Yue Q, Liu M, Nie X, Wu Q, Li J, et al. Quantitative 3.0 T MR spectroscopy reveals decreased creatine concentration in the dorsolateral prefrontal cortex of patients with social anxiety disorder. Plos One. 2012;7(10):e48105. doi: 10.1371/journal. pone.0048105. PubMed PMID: 23110183. PubMed PMCID: PMC3479123.

31. Amenta F, Tayebati SK. Pathways of acetylcholine synthesis, transport and release as targets for treatment of adult-onset cognitive dysfunction. Current Medicinal Chemistry. 2008;15(5):488-98. doi: 10.2174/092986708783503203. PubMed PMID: 18289004.

32. Sarter M, Parikh V. Choline transporters, cholinergic transmission and cognition. Nature Reviews Neuroscience. 2005;6(1):48-56. doi: 10.1038/nrn1588. PubMed PMID: 15611726.

33. Ferguson KJ, MacLullich AM, Marshall I, Deary $\mathrm{IJ}$, et al. Magnetic resonance spectroscopy and cognitive function in healthy elderly men. Brain. 2002;125(12):2743-9. doi: 10.1093/brain/awf278. PubMed PMID: 12429601. 